Research Article; Received: December 25, 2020; Accepted: December 14, 2021

\title{
EXPONENTIAL STABILITY OF A TIMOSHENKO TYPE THERMOELASTIC SYSTEM WITH GURTIN-PIPKIN THERMAL LAW AND FRICTIONAL DAMPING
}

\author{
Abdelfeteh FAREH
}

Laboratory of Operator Theory and PDE's, University of El Oued, ALGERIA

\begin{abstract}
In this paper we consider a linear thermoelastic system of Timoshenko type where the heat conduction is given by the linearized law of GurtinPipkin. An existence and uniqueness result is proved by the use of a semigroup approach. We establish an exponential stability result without any assumption on the wave speeds once here we have a fully damped system.
\end{abstract}

\section{INTRODUCTION}

In the present paper we investigate the well-posedness and the asymptotic behavior of the following Timoshenko type system

$$
\begin{cases}\rho_{1} u_{t t}=\kappa\left(u_{x}+\varphi\right)_{x} & \text { in }(0, \pi) \times \mathbb{R}_{+}, \\ \rho_{2} \varphi_{t t}=b \varphi_{x x}-\kappa\left(u_{x}+\varphi\right)+\delta \theta-\tau \varphi_{t} & \text { in }(0, \pi) \times \mathbb{R}_{+}, \\ c \theta_{t}=-q_{x}-\delta \varphi_{t} & \text { in }(0, \pi) \times \mathbb{R}_{+},\end{cases}
$$

where $u$ is the transverse displacement of a beam of length $\pi, \varphi$ is the rotation angle of filament, $\theta$ is the temperature variation from an equilibrium reference value and $q$ is the heat flux. The coefficients $\rho_{1}, \rho_{2}, c, \kappa, \tau$ are positive and present the mass density, the polar moment of inertia of a cross section, the specific heat constant, the shear modulus and the intensity of the frictional damping respectively, $b=E I$ is the product of Young's modulus of elasticity and the moment of inertia of a cross section, $\beta$ and $\delta$ are coupling constants that are different from zero but their signs does not matter in the analysis.

To render the system (1) determined an additional equation relating $q$ and $\theta$ is needed. In the classical theory of thermoelasticity the constitutive equation for the

2020 Mathematics Subject Classification. 35D35, 74B05, 93D23.

Keywords. Timoshenko system, well-posedness, exponential stability.

arehabdelf@gmail.com

(D) 0000-0003-3522-324X. 
heat flux is expressed through Fourier's law of heat conduction

$$
q=-k \theta_{x}
$$

where $k>0$ represents the coefficient of the thermal conductivity of the material.

In 1921, Timoshenko 32 introduced a shear deformation and a rotational inertia into the derivation of the vibrating beam theory. He modelled the transverse vibrations of a beam by the conservative system

$$
\begin{cases}\rho u_{t t}=\left(K\left(u_{x}-\varphi\right)\right)_{x}, & \text { in }(0, L) \times(0, \infty) \\ I_{\rho} \varphi_{t t}=\left(E I \varphi_{x}\right)_{x}+K\left(u_{x}-\varphi\right), & \text { in }(0, L) \times(0, \infty)\end{cases}
$$

In the last three decades, the system $(3)$ has been intensively studied for possible damping mechanisms. Muñoz Rivera and Racke 25] introduced a thermal damping by coupling system (3) with the classical heat equation. They proved that the system

$$
\left\{\begin{array}{l}
\rho_{1} \varphi_{t t}=k\left(\varphi_{x}+\psi\right)_{x} \\
\rho_{2} \psi_{t t}=b \psi_{x x}-k\left(\varphi_{x}+\psi\right)+\gamma \theta_{x} \\
c \theta_{t}=\kappa \theta_{x x}-\gamma \psi_{t x}
\end{array}\right.
$$

(of course with some boundary and initial conditions), is exponentially stable if and only if

$$
\frac{\rho_{1}}{k}=\frac{\rho_{2}}{b} .
$$

If (5) does not hold Guesmia et al. 17. established a polynomial decay result provided that the initial data are regular enough.

Almeida Junior et al. 11 considered the thermal coupling of the system (3) in shear force

$$
\begin{cases}\rho_{1} \varphi_{t t}-\kappa\left(\varphi_{x}+\psi\right)_{x}+\sigma \theta_{x}=0 & \text { in }(0, L) \times \mathbb{R}_{+}, \\ \rho_{2} \psi_{t t}-b \psi_{x x}+\kappa\left(\varphi_{x}+\psi\right)-\sigma \theta=0 & \text { in }(0, L) \times \mathbb{R}_{+}, \\ \rho_{3} \theta_{t}-\gamma \theta_{x x}+\sigma\left(\varphi_{x}+\psi\right)_{t}=0 & \text { in }(0, L) \times \mathbb{R}_{+},\end{cases}
$$

subjected to either the boundary conditions

$$
\varphi(t, 0)=\varphi(t, L)=\psi(t, 0)=\psi(t, L)=\theta(t, 0)=\theta(t, L)=0,
$$

or

$$
\varphi(t, 0)=\varphi(t, L)=\psi_{x}(t, 0)=\psi_{x}(t, L)=\theta_{x}(t, 0)=\theta_{x}(t, L)=0,
$$

and proved that the solution is exponentially stable if and only if

$$
\chi=\frac{\kappa}{\rho_{1}}-\frac{b}{\rho_{2}}=0 .
$$

Otherwise, when $(9)$ does not hold, the authors showed that the system is polynomially stable with a rate of decay $t^{-1 / 4}$ for the boundary conditions $(7)$ and an optimal rate of decay $t^{-1 / 2}$ for the boundary conditions $(8)$. Recently $1 \overline{8}$ reached the rate $t^{-1 / 2}$ for the boundary conditions $(7)$ and

$$
\varphi_{x}(t, 0)=\varphi_{x}(t, L)=\psi(t, 0)=\psi(t, L)=\theta_{x}(t, 0)=\theta_{x}(t, L)=0 .
$$


Alves et al. [2] improve the results of [1] for the case of different wave speeds and obtained the same rate of decay $t^{-1 / 2}$ independently of the boundary conditions. Later, Alves et al. [3] extended the results of [1] to the non-homogeneous case with the boundary conditions (7). Precisely, they established an exponential stability provided that the non-homogeneous wave speeds satisfy the condition

$$
\frac{\kappa(x)}{\rho_{1}(x)}=\frac{b(x)}{\rho_{2}(x)}, x \in I \subset(0, L)
$$

in an open subinterval $I$ of $(0, L)$. When 10 does not hold they obtained a polynomial stability result with a rate of decay depending on the regularity of the initial data.

Recently, Jorge-Silva and Racke [19] considered (6) with Cattaneo's law and proved that there is non exponential stability no matter if 9 holds which confirms the result of $[10]$.

We recall that the model using Fourier's law (2) leads to a parabolic equation. Consequently, the heat propagates with an infinite speed, that is, any thermal disturbance produced at some point in the body has an instantaneous effect elsewhere in the body. To overcome this physical paradox, many theories were developed. Green and Naghdi 12 14] expanded three new theories based on an entropy equality rather than the entropy inequality. They called them thermoelasticity of type I, type II and type III respectively. In each of these theories the equation for the heat flux is given by a different constitutive assumption. The constitutive equation for the heat flux in the type III theory is given by

$$
q=-f_{1} \alpha_{x}-f_{2} \theta_{x}
$$

where

$$
\alpha=\alpha_{0}(x)+\int_{0}^{t} \theta(x, \tau) d \tau
$$

is the thermal displacement and $f_{1}, f_{2}$ are two positive constants.

In the framework of the thermoelasticity of type III, Messaoudi and Said-Houari 24] considered the following Timoshenko type system

$$
\begin{cases}\rho_{1} \varphi_{t t}-K\left(\varphi_{x}+\psi\right)_{x}=0 & \text { in }(0,1) \times(0,+\infty) \\ \rho_{2} \psi_{t t}-b \psi_{x x}+K\left(\varphi_{x}+\psi\right)+\beta \theta_{x}=0 & \text { in }(0,1) \times(0,+\infty) \\ \rho_{3} \theta_{t t}-\delta \theta_{x x}+\beta \psi_{t t x}+\kappa \theta_{t x x}=0 & \text { in }(0,1) \times(0,+\infty)\end{cases}
$$

and showed that the solution $(\varphi, \psi, \theta)$ decays exponentially provided that $\frac{K}{\rho_{1}}=\frac{b}{\rho_{2}}$. The case of non equal speeds was examined by Messaoudi and Fareh [23. They established a polynomial rate of decay. Fatori et al. [9] show that the optimal rate in this case is $t^{-1 / 2}$. 
Santos and Almeida Júnior 30$]$ extended the results of 23 24 to the Timoshenko system with thermoelastic effect acting on a shear force

$$
\begin{cases}\rho_{1} \varphi_{t t}-K\left(\varphi_{x}+\psi\right)_{x}+\sigma \theta_{t x}=0 & \text { in }(0, L) \times(0,+\infty), \\ \rho_{2} \psi_{t t}-b \psi_{x x}+K\left(\varphi_{x}+\psi\right)-\sigma \theta_{t}=0 & \text { in }(0, L) \times(0,+\infty), \\ \rho_{3} \theta_{t t}-\delta \theta_{x x}+\sigma\left(\varphi_{x}+\psi\right)_{t}-\gamma \theta_{t x x}=0 & \text { in }(0, L) \times(0,+\infty) .\end{cases}
$$

The second theory proposed to overcome the paradox of infinite speed was developed by Lord and Shulman [21]. They suggested to replace Fourier's law (2) by Cattaneo's one

$$
\tau_{0} q_{t}+q+k \theta_{x}=0
$$

where the positive constant $\tau_{0}$ represents the time lag in the response of the heat flux to the temperature gradient and is referred to as the thermal relaxation time. According to this theory, the system becomes fully hyperbolic, as a result the heat propagates with a finite speed and is viewed as a wave-like propagation rather than a diffusion phenomenon. A wave-like thermal disturbance is referred to as a second sound (where the first sound being the usual sound) and a nonclassical theory predicting the occurrence of such disturbances are known as thermoelasticity with finite wave speeds or second sound thermoelasticity.

Fernández Sare and Racke [10 considered the following Timoshenko type system with second sound thermoelasticity

$$
\left\{\begin{array}{l}
\rho_{1} \varphi_{t t}-k\left(\varphi_{x}+\psi\right)_{x}=0 \\
\rho_{2} \psi_{t t}-b \psi_{x x}+k\left(\varphi_{x}+\psi\right)+\delta \theta_{x}=0 \\
\rho_{3} \theta_{t}+\gamma q_{x}+\delta \psi_{t x}=0 \\
\tau_{0} q_{t}+q+\kappa \theta_{x}=0
\end{array}\right.
$$

and proved that the solution of $(11)$ is no longer exponentially stable even if $\frac{\rho_{1}}{k}=\frac{\rho_{2}}{b}$. However, the incorporation of the frictional damping $\mu \varphi_{t}$ into the first equation of (11) produces an exponential stability independently of the wave speeds [22].

Santos et al. 31 introduced the stability number

$$
\chi_{0}=\left(\tau-\frac{\rho_{1}}{\rho_{3} \kappa}\right)\left(\rho_{2}-\frac{b \rho_{1}}{\kappa}\right)-\frac{\tau \rho_{1} \delta^{2}}{\kappa \rho_{3}},
$$

and proved that the solution of $(11)$ is exponentially stable provided that $\chi_{0}=0$.

It is worth noting that the type III thermoelasticity and the second sound thermoelasticity are unable to describe the memory effect which reigns in some materials, particularly at a low temperature. This fact leads to the look for a more general constitutive assumption relating the heat flux to the thermal memory. Gurtin and Pipkin [16] assumed that the heat flux depends on the integrated history of the temperature gradient, and established a general nonlinear theory for which thermal disturbances propagate with a finite speed. In accordance with this theory, the linearized constitutive equation for $q$ is given by

$$
q=-\int_{-\infty}^{t} k(t-s) \theta_{x}(x, s) d s
$$


where $k(s)$ is the heat conductivity relaxation kernel. The presence of the convolution term 12 renders the Timoshenko system coupled with the heat equation into a fully hyperbolic system, which allows the heat to propagate with a finite speed and admits to describe the memory effect of the heat conduction.

In the context of Gurtin-Pipkin theory Pata and Vuk 26 studied the linear thermoelastic system

$$
\left\{\begin{array}{c}
u_{t t}(x, t)=u_{x x}(x, t)-\theta_{x}(x, t) \\
\theta_{t}(x, t)=-u_{t x}(x, t)-q_{x}(x, t)
\end{array}\right.
$$

where the heat flux $q$ is given by (12). They proved, under some assumptions on $\mu(s)=-k^{\prime}(s)$, that the solution of the system decays exponentially. Fatori and Muñoz Rivera [8] considered the system

$$
\left\{\begin{array}{c}
u_{t t}-a u_{x x}+\alpha \theta_{x}=0 \text { in }(0, L) \times \mathbb{R}_{+} \\
\theta_{t}-k * \theta_{x x}+\alpha u_{x t}=0 \text { in }(0, L) \times \mathbb{R}_{+},
\end{array}\right.
$$

where

$$
\left(k * \theta_{x x}\right)(t)=\int_{0}^{t} k(t-\tau) \theta_{x x}(\tau) d \tau,
$$

and established an exponential decay result provided that the kernel $k$ is positive definite and decays exponentially.

Concerning Timoshenko systems coupled with the heat equation in the framework of Gurtin-Pipkin's theory, Dell'Oro an Pata [7] analyzed the following system

$$
\left\{\begin{array}{l}
\rho_{1} \varphi_{t t}-\kappa\left(\varphi_{x}+\psi\right)_{x}=0 \\
\rho_{2} \psi_{t t}-b \psi_{x x}+\kappa\left(\varphi_{x}+\psi\right)+\delta \theta_{x}=0 \\
\rho_{3} \theta_{t}-\frac{1}{\beta} \int_{0}^{\infty} g(s) \theta_{x x}(t-s) d s+\delta \psi_{t x}=0
\end{array}\right.
$$

and proved that the semigroup associated with the solution of the system 13 is exponentially stable if and only if

$$
\chi_{g}=\left[\frac{\rho_{1}}{\rho_{3} \kappa}-\frac{\beta}{g(0)}\right]\left[\frac{\rho_{1}}{\kappa}-\frac{\rho_{2}}{b}\right]-\frac{\beta}{g(0)} \frac{\rho_{1} \delta^{2}}{\rho_{3} \kappa b}=0 .
$$

Closely related to Timoshenko's beam theory, Raposo 29 investigated the laminated Timoshenko system

$$
\begin{cases}\rho_{1} u_{t t}-\kappa\left(u_{x}-\psi\right)_{x}+\alpha u_{t}=0 & \text { in }(0, L) \times \mathbb{R}_{+} \\ \rho_{2}(s-\psi)_{t t}-b(s-\psi)_{x x}+\kappa\left(\psi-u_{x}\right)+\beta(s-\psi)_{t}=0 & \text { in }(0, L) \times \mathbb{R}_{+} \\ \rho_{2} s_{t t}-b s_{x x}+3 \kappa\left(\psi-u_{x}\right)+4 \delta s+4 \gamma s_{t}=0 & \text { in }(0, L) \times \mathbb{R}_{+}\end{cases}
$$

and obtained an exponential stability result. Regarding the damping by the heat conduction, Liu and Zhao 20 showed that the laminated beam coupled with the heat equation modelled via Fourier's law of the heat conduction is exponentially stable provided that the wave speeds are equal. Apalara 4 obtained the same result by coupling the laminated beam with the heat equation moddeled via Cattaneo's 
law, provided that the equal wave speeds is replaced by a relation between the coefficients of the system. Choucha et al. [5] added a distributed delay and proved the exponential and the polynomial stability for the equal and the non-equal wave speeds respectively. They also kept the same results in the presence of a viscoelastic damping and a distributed delay [6].

In view of the aforementioned studies we can summarized the stability results for Timoshenko systems coupled with thermal effects as follows:

i) A fully damped Timoshenko system with parabolic thermal effects is exponentially stable regardless any restriction on the wave speeds.

ii) A Timoshenko system damped only by thermal effects is exponentially stable if and only if the coefficients of the system satisfy a stability condition (equal wave speeds, in the case of the classical parabolic heat equation).

To the best of my knowledge there is no results concerning the fully damped Timoshenko system with hyperbolic thermal dissipation. One can expected that this leads to an exponential stability. In the present paper we give a positive answer to this concern.

It should be noted here, that replacing the parabolic heat conduction by a hyperbolic type one is not obviously profitable, first, because the system becomes fully hyperbolic and therefore it loses the exponential decay reached with one dissipation when (5) holds, (see [10,28]), secondly, because the dissipative effects due to the hyperbolic type heat conduction are generally weaker than those induced by Fourier's law.

In the present paper we consider the fully damped case of (13) and prove the exponential stability of the solution without any condition. The importance of our result manifested from the fact that the case of equal speeds is purely mathematical, since it is physically never satisfied [15]. Therefore, the stability result obtained without any restriction on the coefficients is more realistic than that obtained with a stability condition.

Note that the presence of the convolution term in the constitutive equation for $q$ renders the family operators mapping the initial value $\left(u_{0}, u_{1}, \varphi_{0}, \varphi_{1}, \theta_{0}\right)$ into the solution $(u, \varphi, \theta)$ not match the semigroup properties. This is due to the fact that the solution value of $\theta$ at time $t$ depends on the whole function up to time $t$.

In order to overcome this difficulty we introduce the new variables

$$
\theta^{t}(x, s)=\theta(x, t-s), s \geq 0
$$

and

$$
\eta(x, s)=\eta^{t}(x, s)=\int_{0}^{s} \theta^{t}(x, \tau) d \tau, s \geq 0,
$$

which denote the past history and the summed past history of $\theta$ up to $t$, respectively.

Clearly $\eta^{t}(x, s)$ satisfies the boundary conditions

$$
\eta(0, s)=\eta(\pi, s)=0
$$


EXPONENTIAL STABILITY OF TIMOSHENKO SYSTEM WITH G-P THERMAL LAW 101

Moreover, we assume that $k(\infty)=0$ and $\eta(x, 0)=\lim _{s \longrightarrow 0^{+}} \eta^{t}(x, s)=0$, then

$$
q=-\int_{-\infty}^{t} k(t-s) \theta_{x}(x, s) d s=\int_{0}^{\infty} k^{\prime}(s) \eta_{x}^{t}(x, s) d s .
$$

Further, we have

$$
\eta_{t}(x, s)=\theta-\eta_{s}(x, s) .
$$

Setting $\mu(s)=-k^{\prime}(s)$, the system (1) and equations (12), (15) become

$$
\begin{cases}\rho_{1} u_{t t}=\kappa\left(u_{x x}+\varphi_{x}\right)-\beta \theta_{x} & \text { in }(0, \pi) \times \mathbb{R}_{+}, \\ \rho_{2} \varphi_{t t}=b \varphi_{x x}-\kappa\left(u_{x}+\varphi\right)+\delta \theta-\tau \varphi_{t} & \text { in }(0, \pi) \times \mathbb{R}_{+}, \\ c \theta_{t}=\int_{0}^{\infty} \mu(s) \eta_{x x}^{t}(s) d s-\beta u_{x t}-\delta \varphi_{t} & \text { in }(0, \pi) \times \mathbb{R}_{+}, \\ \eta_{t}^{t}(s)=\theta-\eta_{s}^{t}(s) & \text { in }(0, \pi) \times \mathbb{R}_{+} \times \mathbb{R}_{+} .\end{cases}
$$

The system 16 is complemented with the boundary conditions

$$
\begin{aligned}
& u(0, t)=u(\pi, t)=\varphi_{x}(0, t)=\varphi_{x}(\pi, t)=\theta(0, t)=\theta(\pi, t)=0, \\
& \eta(0, s)=\eta(\pi, s)=0, \forall t \in \mathbb{R}^{+}, \eta(x, 0)=0, \forall x \in(0, \pi),
\end{aligned}
$$

and the initial data

$$
\begin{gathered}
u(x, 0)=u_{0}(x), u_{t}(x, 0)=u_{1}(x), \varphi(x, 0)=\varphi_{0}(x), \\
\varphi_{t}(x, 0)=\varphi_{1}(x), \theta(x, 0)=\theta_{0}(x), \eta^{0}(x, s)=\eta_{0}(x, s) .
\end{gathered}
$$

Regarding the memory kernel $\mu$, we assume the following set of hypotheses:

(h1) $\mu \in C\left(\mathbb{R}^{+}\right) \cap L^{1}\left(\mathbb{R}^{+}\right)$,

(h2) $\mu(s) \geq 0, \mu^{\prime}(s) \leq 0 \quad \forall s \geq 0$,

(h3) $\int_{0}^{\infty} \mu(s) d s=k_{0}>0$,

(h4) there exists $\xi>0$, such that $\mu^{\prime}(s) \leq-\xi \mu(s), \forall s \geq 0$.

The rest of the paper is organized as follows: in Section 2, we introduce some functional preliminaries. Section 3 is devoted to the proof of an existence and uniqueness result. In Section 4, we state and prove our stability result.

\section{Functional Setting}

Let $A=-D^{2}$ be the operator defined over $L^{2}(0, \pi)$. It is well known that the operator $A$ with the Dirichlet boundary conditions is a self-adjoint and positive operator with domain $D(A)=H^{2} \cap H_{0}^{1}$. Thus, it is possible to define the powers $A^{\alpha}$ of $A$ for $\alpha \in \mathbb{R}$, and the Hilbert space $V_{\alpha}=D\left(A^{\alpha / 2}\right)$ endowed with the inner product

$$
\langle u, v\rangle_{\alpha}=\left\langle A^{\alpha / 2} u, A^{\alpha / 2} v\right\rangle
$$

and the associated norm denoted by $\|u\|_{\alpha}$. In particular, $V_{0}=L^{2}, V_{-1}=H^{-1}$, $V_{1}=H_{0}^{1}$ and

$$
\left\langle A^{1 / 2} u, A^{1 / 2} v\right\rangle=\langle D u, D v\rangle, \forall u, v \in H_{0}^{1} .
$$

For $\alpha_{1}>\alpha_{2}$ the injection $V_{\alpha_{1}} \hookrightarrow V_{\alpha_{2}}$ is continuous. 
Furthermore, we introduce the weighted Hilbert space

$$
\mathcal{M}_{1}=L_{\mu}^{2}\left((0,+\infty) ; H_{0}^{1}(0, \pi)\right)
$$

with the inner product

$$
\langle\eta, \zeta\rangle_{\mathcal{M}_{1}}=\int_{0}^{\infty} \mu(s)\langle\eta(s), \zeta(s)\rangle_{1} d s
$$

and the norm

$$
\|\eta\|_{\mathcal{M}_{1}}^{2}=\int_{0}^{\infty} \mu(s)\|D \eta(s)\|^{2} d s .
$$

We shall also need to define the spaces

$$
\mathcal{M}_{0}=L_{\mu}^{2}\left((0,+\infty) ; L^{2}(0, \pi)\right)
$$

and

$$
\begin{aligned}
\mathcal{K} & =H_{\mu}^{1}\left((0,+\infty) ; H_{0}^{1}(0, \pi)\right) \\
& =\left\{\eta / \eta, \eta_{s} \in \mathcal{M}_{1}\right\} .
\end{aligned}
$$

The following lemma will be useful in the proof of our main result.

Lemma 1. Let $v \in L^{2}(0, \pi)$ be given and

$$
\bar{v}=\frac{1}{\pi} \int_{0}^{\pi} v(x) d x
$$

the mean value of $v$. Then,

$$
\|D v\|_{-1}=\|v-\bar{v}\| .
$$

Proof. We have

$$
\|D v\|_{-1}=\sup _{\|D \psi\|=1}|\langle D v, \psi\rangle|=\sup _{\|D \psi\|=1}|\langle v, D \psi\rangle| \leq\|v\| .
$$

Let $\psi(x)=\frac{1}{\|v\|} \int_{0}^{x} v(y) d y$, then $\|D \psi\|=1$ and

$$
|\langle D v, \psi\rangle|=\|v\| \leq\|D v\|_{-1} .
$$

Therefore,

$$
\|D v\|_{-1}=\|v\| .
$$

Suppose that $\bar{v}=0$, then

$$
\|D v\|_{-1}=\|v-\bar{v}\| .
$$

If $\bar{v} \neq 0$, then

$$
\|D v\|_{-1}=\|D(v-\bar{v})\|_{-1}=\|v-\bar{v}\| .
$$




\section{Well Posedness}

In this section we prove that the problem determined by $(16)-18$ has a unique solution. The main tools of the proof are the Lumer-Phillips and the Lax-Milgram theorems. First we need to rewrite the problem in the semigroups setting.

Let $\mathcal{H}$ be the Hilbert space

$$
\mathcal{H}=H_{0}^{1} \times L^{2} \times H_{*}^{1} \times L_{*}^{2} \times L^{2} \times \mathcal{M}_{1}
$$

endowed with the inner product

$$
\begin{aligned}
\left\langle U, U^{*}\right\rangle= & \kappa \int_{0}^{\pi}\left(u_{x}+\varphi\right)\left(u_{x}^{*}+\varphi^{*}\right) d x+\rho_{1} \int_{0}^{\pi} v v^{*} d x+b \int_{0}^{\pi} \varphi_{x} \varphi_{x}^{*} d x \\
& +\rho_{2} \int_{0}^{\pi} \phi \phi^{*} d x+c \int_{0}^{\pi} \theta \theta^{*} d x+\int_{0}^{\infty} \int_{0}^{\pi} \mu(s) \eta_{x}(s) \eta_{x}^{*}(s) d x d s
\end{aligned}
$$

and the associated norm

$$
\|U\|_{\mathcal{H}}^{2}=\kappa\left\|u_{x}+\varphi\right\|^{2}+\rho_{1}\|v\|^{2}+b\left\|\varphi_{x}\right\|^{2}+\rho_{2}\|\phi\|^{2}+c\|\theta\|^{2}+\|\eta\|_{\mathcal{M}_{1}}^{2} .
$$

We note that by virtue of the inequalities

$$
\begin{aligned}
u_{x}^{2} & \leq 2\left(u_{x}+\varphi\right)^{2}+2 \varphi^{2}, \\
\left(u_{x}+\varphi\right)^{2} & \leq 2 u_{x}^{2}+2 \varphi^{2}
\end{aligned}
$$

the above norm in $\mathcal{H}$ is equivalent to the usual norm. Therefore, we use either of the norms indifferently.

To rewrite the system $(16)$ in the semigroup setting we introduce the new variables $v=u_{t}$ and $\phi=\varphi_{t}$, then the system (16) becomes

$$
\left\{\begin{array}{l}
u_{t}=v \\
v_{t}=\frac{\kappa}{\rho_{1}}\left(u_{x x}(x, t)+\varphi_{x}(x, t)\right)-\frac{\beta}{\rho_{1}} \theta_{x}(x, t) \\
\varphi_{t}=\phi \\
\phi_{t}=\frac{b}{\rho_{2}} \varphi_{x x}(x, t)-\frac{\kappa}{\rho_{2}}\left(u_{x}(x, t)+\varphi(x, t)\right)+\frac{\delta}{\rho_{2}} \theta(x, t)-\frac{\tau}{\rho_{2}} \phi(x, t) \\
\theta_{t}(x, t)=\frac{1}{c} \int_{0}^{\infty} \mu(s) \eta_{x x}^{t}(x, s) d s-\frac{\beta}{c} v_{x}(x, t)-\frac{\delta}{c} \phi(x, t) \\
\eta_{t}^{t}(x, s)=\theta(x, t)-\eta_{t}^{t}(x, s)
\end{array}\right.
$$

and the problem $(16)-18)$ rewritten

$$
\left\{\begin{array}{c}
\frac{d}{d t} U=\mathcal{A} U, t>0, \\
U(0)=U_{0},
\end{array}\right.
$$


where, $\mathcal{A}$ is the operator defined by

$$
\mathcal{A} U=\left(\begin{array}{c}
v \\
\frac{\kappa}{\rho_{1}} u_{x x}+\frac{\kappa}{\rho_{1}} \varphi_{x}-\frac{\beta}{\rho_{1}} \theta_{x} \\
\phi \\
\frac{b}{\rho_{2}} \varphi_{x x}-\frac{\kappa}{\rho_{2}} u_{x}-\frac{\kappa}{\rho_{2}} \varphi+\frac{\delta}{\rho_{2}} \theta-\frac{\tau}{\rho_{2}} \phi \\
\frac{1}{c} \int_{0}^{\infty} \mu(s) \eta_{x x}(s) d s-\frac{\beta}{c} v_{x}-\frac{\delta}{c} \phi \\
\theta-\eta_{s}
\end{array}\right)
$$

with domain

$$
D(\mathcal{A}):=\left\{\begin{array}{c}
U \in \mathcal{H} ; u, \varphi \in H^{2}, v, \theta \in H_{0}^{1}, \phi \in H_{*}^{1}, \eta \in H_{\mu}^{1}\left((0,+\infty) ; H_{0}^{1}\right), \\
\int_{0}^{\infty} \mu(s) \eta_{x x}(s) d s \in L^{2}, \eta(0)=0
\end{array}\right\} .
$$

Before stating the main result of this section let us recall the following theorems.

Theorem 1. (Lumer-Phillips) [27]33] Let $\mathcal{A}: D(\mathcal{A}) \subset \mathcal{H} \longrightarrow \mathcal{H}$ be a densely defined operator. Then $\mathcal{A}$ generates a $C_{0}$-semigroup of contractions on $\mathcal{H}$ if and only if

i) $\mathcal{A}$ is dissipative;

ii) there exists a constant $\lambda>0$ such that $\lambda I-\mathcal{A}$ is onto.

Theorem 2. [33] Let $\mathcal{A}: D(\mathcal{A}) \subset \mathcal{H} \longrightarrow \mathcal{H}$ be the infinitesimal generator of a $C_{0}$-semigroup $\{S(t) ; t \geq 0\}$. Then, for each $\xi \in D(\mathcal{A})$ and each $t \geq 0$, we have $S(t) \xi \in D(\mathcal{A})$ and the mapping

$$
\begin{aligned}
& S:[0,+\infty[\longrightarrow \mathcal{H} \\
& t \longrightarrow S(t) \xi
\end{aligned}
$$

is of class $C^{1}$ on $[0,+\infty[$ and satisfies

$$
\frac{d}{d t}(S(t) \xi)=\mathcal{A} S(t) \xi=S(t) \mathcal{A} \xi .
$$

Our main result reads as follows:

Theorem 3. Suppose that $\mu$ satisfies the hypotheses (h1)-(h4), then for any $U_{0}=$ $\left(u_{0}, u_{1}, \varphi_{0}, \varphi_{1}, \theta_{0}, \eta_{0}\right)^{T} \in \mathcal{H}$ the problem (20) has a unique solution $U \in C((0,+\infty) ; \mathcal{H})$. Moreover, if $U_{0}=\left(u_{0}, u_{1}, \varphi_{0}, \varphi_{1}, \theta_{0}, \eta_{0}\right)^{T} \in D(\mathcal{A})$ then the solution $U$ satisfies

$$
U \in C((0,+\infty) ; D(\mathcal{A})) \cap C^{1}((0,+\infty) ; \mathcal{H}) .
$$

Proof. First, we prove that $\mathcal{A}$ is dissipative. Indeed, for every $U \in D(\mathcal{A})$ we have

$$
\begin{gathered}
\langle\mathcal{A} U, U\rangle=\kappa \int_{0}^{\pi}\left(v_{x}+\phi\right)\left(u_{x}+\varphi\right) d x+\int_{0}^{\pi}\left(\kappa u_{x x}+\kappa \varphi_{x}-\beta \theta_{x}\right) v d x+b \int_{0}^{\pi} \phi_{x} \varphi_{x} d x \\
+\int_{0}^{\pi}\left(b \varphi_{x x}-\kappa u_{x}-\kappa \varphi+\delta \theta-\tau \phi\right) \phi d x
\end{gathered}
$$


EXPONENTIAL STABILITY OF TIMOSHENKO SYSTEM WITH G-P THERMAL LAW 105

$$
\begin{aligned}
& +\int_{0}^{\pi}\left(\int_{0}^{\infty} \mu(s) \eta_{x x}^{t}(s) d s-\beta v_{x}-\delta \phi\right) \theta d x \\
& \quad+\int_{0}^{\pi} \int_{0}^{\infty} \mu(s)\left(\theta_{x}-\eta_{x s}^{t}\right) \eta_{x}(s) d s d x \\
& =-\tau \int_{0}^{\pi} \phi^{2} d x-\frac{1}{2} \int_{0}^{\infty} \mu(s) \frac{d}{d s}\left\|\eta_{x}(s)\right\|^{2} d s .
\end{aligned}
$$

For the second term in the right-hand side, we have

$$
\int_{0}^{\infty} \mu(s) \frac{d}{d s}\left\|\eta_{x}(s)\right\|^{2} d s=\left.\mu(s)\left\|\eta_{x}(s)\right\|^{2}\right|_{0} ^{\infty}-\int_{0}^{\infty} \mu^{\prime}(s)\left\|\eta_{x}(s)\right\|^{2} d s .
$$

Since $\mu(s)\left\|\eta_{x}(s)\right\|^{2}$ and $\mu(s)\left\|\eta_{x s}(s)\right\|^{2}$ belong to $L^{1}\left(\mathbb{R}^{+}\right)$and $\eta_{x}(0)=0$, hence

$$
\begin{aligned}
\lim _{s \rightarrow 0} \mu(s)\left\|\eta_{x}(s)\right\|^{2} & =\lim _{s \rightarrow 0} \mu(s)\left\|\int_{0}^{s} \eta_{x s}(\tau) d \tau\right\|^{2}, \\
& \leq \limsup _{s \rightarrow 0}\left(\int_{0}^{s} \mu(s)^{1 / 2}\left\|\eta_{x s}(\tau)\right\| d \tau\right)^{2} .
\end{aligned}
$$

The Cauchy-Schwarz inequality, leads to

$$
\lim _{s \rightarrow 0} \mu(s)\left\|\eta_{x}(s)\right\|^{2} \leq \limsup _{s \rightarrow 0} s \int_{0}^{s} \mu(\tau)\left\|\eta_{x s}(\tau)\right\|^{2} d \tau=0 .
$$

Therefore,

$$
\int_{0}^{\infty} \mu(s) \frac{d}{d s}\left\|\eta_{x}(s)\right\|^{2} d s=\lim _{s \rightarrow \infty} \mu(s)\left\|\eta_{x}(s)\right\|^{2}-\int_{0}^{\infty} \mu^{\prime}(s)\left\|\eta_{x}(s)\right\|^{2} d s .
$$

The left-hand side of the last equation is bounded, and from (h2) both terms on the right-hand side are positive. Then, the limit in the right hand side exists and is finite, and therefore equals zero. Thus,

$$
\langle\mathcal{A} U, U\rangle=-\tau \int_{0}^{\pi} \phi^{2} d x+\frac{1}{2} \int_{0}^{\infty} \mu^{\prime}(s)\left\|\eta_{x}(s)\right\|^{2} d s \leq 0,
$$

which proves the dissipativeness of $\mathcal{A}$. Next, we show that $\mathcal{A}$ is maximal. Let $U^{*}=\left(u^{*}, v^{*}, \varphi^{*}, \phi^{*}, \theta^{*}, \eta^{*}\right)^{T} \in \mathcal{H}$, and find $U=(u, v, \varphi, \phi, \theta, \eta)^{T} \in D(\mathcal{A})$ such that

which reads in components

$$
(I-\mathcal{A}) U=U^{*}
$$

$$
\begin{gathered}
u-v=u^{*} \\
\rho v-\kappa u_{x x}-\kappa \varphi_{x}+\beta \theta_{x}=\rho_{1} v^{*}, \\
\varphi-\phi=\varphi^{*}, \\
\left(\rho_{2}+\tau\right) \phi-b \varphi_{x x}+\kappa u_{x}+\kappa \varphi-\delta \theta=\rho_{2} \phi^{*}, \\
c \theta-\int_{0}^{\infty} \mu(s) \eta_{x x}^{t}(s) d s+\beta v_{x}+\delta \phi=c \theta^{*}, \\
\eta-\theta+\eta_{s}=\eta^{*} .
\end{gathered}
$$


Solving equation 27] gives

$$
\eta(s)=\left(1-e^{-s}\right) \theta+\int_{0}^{s} e^{y-s} \eta^{*}(y) d y .
$$

Substituting (22), (24) and (28) into (23), 25) and (26) we get

$$
\left\{\begin{array}{l}
\kappa u_{x x}+\kappa \varphi_{x}-\beta \theta_{x}-\rho_{1} u=-\rho_{1}\left(u^{*}+v^{*}\right), \\
b \varphi_{x x}-\kappa u_{x}-\left(\kappa+\rho_{2}+\tau\right) \varphi+\delta \theta=-\left(\rho_{2}+\tau\right) \varphi^{*}-\rho_{2} \phi^{*}, \\
c_{\mu} \theta_{x x}-c \theta-\beta u_{x}-\delta \varphi=-\left(c \theta^{*}+\beta u^{*}+\delta \varphi^{*}\right)-\int_{0}^{\infty} \mu(s)\left(\int_{0}^{s} e^{y-s} \eta_{x x}^{*}(y) d y\right) d s
\end{array}\right.
$$

where,

$$
c_{\mu}=\int_{0}^{\infty} \mu(s)\left(1-e^{-s}\right) d s
$$

is a positive constant. The last term in the right-hand side of the third equation of 29. belongs to $H^{-1}$. Indeed, let $\psi \in H_{0}^{1}$ such that $\left\|\psi_{x}\right\| \leq 1$, then

$$
\begin{aligned}
\left|\left\langle\int_{0}^{\infty} \mu(s)\left(\int_{0}^{s} e^{y-s} \eta_{x x}^{*}(y) d y\right) d s, \psi\right\rangle\right| & =\left|\left\langle\int_{0}^{\infty} \mu(s)\left(\int_{0}^{s} e^{y-s} \eta_{x}^{*}(y) d y\right) d s, \psi_{x}\right\rangle\right| \\
& \leq \int_{0}^{\infty} \mu(s) e^{-s}\left(\int_{0}^{s} e^{y}\left\|\eta_{x}^{*}(y)\right\| d y\right) d s \\
& =\int_{0}^{\infty} e^{y}\left\|\eta_{x}^{*}(y)\right\| \int_{y}^{\infty} \mu(s) e^{-s} d s d y \\
& \leq \int_{0}^{\infty} \mu(y) e^{y}\left\|\eta_{x}^{*}(y)\right\| \int_{y}^{\infty} e^{-s} d s d y \\
& =\int_{0}^{\infty} \mu(y)\left\|\eta_{x}^{*}(y)\right\| d y<\infty .
\end{aligned}
$$

At this point we multiply the equations $(29,1,29)_{2}$ and 29$)_{3}$ by $\widetilde{u}, \widetilde{\varphi}$ and $\widetilde{\theta}$ respectively, integrating over $(0, \pi)$ and summing up, we obtain

$$
B(U, \widetilde{U})=L(\widetilde{U}),
$$

where

$$
\begin{aligned}
B(U, \widetilde{U}) & :=\kappa \int_{0}^{\pi} u_{x} \widetilde{u}_{x} d x-\kappa \int_{0}^{\pi} \varphi_{x} \widetilde{u} d x+\beta \int_{0}^{\pi} \theta_{x} \widetilde{u} d x+\rho_{1} \int_{0}^{\pi} u \widetilde{u} d x \\
& +b \int_{0}^{\pi} \varphi_{x} \widetilde{\varphi}_{x} d x+\kappa \int_{0}^{\pi} u_{x} \widetilde{\varphi} d x+\left(\kappa+\rho_{2}+\tau\right) \int_{0}^{\pi} \varphi \widetilde{\varphi} d x \\
& -\delta \int_{0}^{\pi} \theta \widetilde{\varphi} d x+c_{\mu} \int_{0}^{\pi} \theta_{x} \widetilde{\theta}_{x} d x+c \int_{0}^{\pi} \theta \widetilde{\theta} d x+\beta \int_{0}^{\pi} u_{x} \widetilde{\theta} d x+\delta \int_{0}^{\pi} \varphi \widetilde{\theta} d x,
\end{aligned}
$$


and

$$
\begin{gathered}
L(\widetilde{U}):=\rho_{1} \int_{0}^{\pi}\left(u^{*}+v^{*}\right) \widetilde{u} d x+\left(\rho_{2}+\tau\right) \int_{0}^{\pi} \varphi^{*} \widetilde{\varphi} d x-\rho_{2} \int_{0}^{\pi} \phi^{*} \widetilde{\varphi} d x \\
+\int_{0}^{\pi}\left(c \theta^{*}+\beta u^{*}+\delta \varphi^{*}\right) \widetilde{\theta} d x+\int_{0}^{\pi} \widetilde{\theta} \int_{0}^{\infty} \mu(s)\left(\int_{0}^{s} e^{y-s} \eta_{x x}^{*}(y) d y\right) d s d x .
\end{gathered}
$$

Clearly, $B(\cdot, \cdot)$ is a bounded bilinear form over $\mathcal{W}=H_{0}^{1} \times H_{*}^{1} \times H_{0}^{1}$ and $L$ is a bounded linear form. Furthermore, we have

$$
\begin{aligned}
B(U, U)= & \kappa \int_{0}^{\pi} u_{x}^{2} d x-\kappa \int_{0}^{\pi} \varphi_{x} u d x+\beta \int_{0}^{\pi} \theta_{x} u d x+\rho_{1} \int_{0}^{\pi} u^{2} d x+b \int_{0}^{\pi} \varphi_{x}^{2} d x \\
& +\kappa \int_{0}^{\pi} u_{x} \varphi d x+\left(\kappa+\rho_{2}+\tau\right) \int_{0}^{\pi} \varphi^{2} d x-\delta \int_{0}^{\pi} \theta \varphi d x+c_{\mu} \int_{0}^{\pi} \theta_{x}^{2} d x \\
& +c \int_{0}^{\pi} \theta^{2} d x+\beta \int_{0}^{\pi} u_{x} \theta d x+\delta \int_{0}^{\pi} \varphi \theta d x, \\
B(U, U) & =\kappa \int_{0}^{\pi}\left(u_{x}+\varphi\right)^{2} d x+\rho_{1} \int_{0}^{\pi} u^{2} d x+b \int_{0}^{\pi} \varphi_{x}^{2} d x \\
& +\left(\rho_{2}+\tau\right) \int_{0}^{\pi} \varphi^{2} d x+c_{\mu} \int_{0}^{\pi} \theta_{x}^{2} d x+c \int_{0}^{\pi} \theta^{2} d x .
\end{aligned}
$$

Therefore, there exists a positive constant $\alpha$ such that

$$
B(U, U) \geq \alpha\|U\|^{2} .
$$

Thus, $B(\cdot, \cdot)$ is coercive and by means of the Lax-Milgram theorem, the problem (30) has a unique solution

$$
(u, \varphi, \theta) \in \mathcal{W} .
$$

Moreover, taking $(\widetilde{u}, \widetilde{\varphi}, \widetilde{\theta})=(\widetilde{u}, 0,0)$ in 300 we get

$$
\kappa \int_{0}^{\pi} u_{x} \widetilde{u}_{x} d x=\int_{0}^{\pi}\left(\kappa \varphi_{x}-\beta \theta_{x}-\rho_{1} u+\rho_{1}\left(u^{*}+v^{*}\right)\right) \widetilde{u} d x, \forall \widetilde{u} \in H_{0}^{1} .
$$

Using standard arguments of elliptic equations we infer that

$$
u \in H^{2}(0, \pi) \cap H_{0}^{1}(0, \pi),
$$

with

$$
\kappa u_{x x}=-\kappa \varphi_{x}+\beta \theta_{x}+\rho_{1} u-\rho_{1}\left(u^{*}+v^{*}\right),
$$

which solves $29 p_{1}$. Similarly, by choosing $(\widetilde{u}, \widetilde{\varphi}, \widetilde{\theta})=(0, \widetilde{\varphi}, 0)$, we obtain $b \int_{0}^{\pi} \varphi_{x} \widetilde{\varphi}_{x} d x=-\int_{0}^{\pi}\left(\kappa\left(u_{x}+\varphi\right)+\left(\rho_{2}+\tau\right)\left(\varphi-\varphi^{*}\right)-\delta \theta-\rho_{2} \phi^{*}\right) \widetilde{\varphi} d x, \forall \widetilde{\varphi} \in H_{*}^{1}$. 
Let $\Psi \in H_{0}^{1}(0, \pi)$ and set

$$
\widetilde{\Psi}(x, t)=\Psi(x, t)-\int_{0}^{\pi} \Psi(x, t) d x .
$$

Clearly $\widetilde{\Psi} \in H_{*}^{1}(0, \pi)$. Plugging $\widetilde{\Psi}$ in 31 and recalling that

$$
\kappa\left(u_{x}+\varphi\right)+\left(\rho_{2}+\tau\right)\left(\varphi-\varphi^{*}\right)-\delta \theta-\rho_{2} \phi^{*} \in L_{*}^{2}(0, \pi),
$$

we arrive at

$b \int_{0}^{\pi} \varphi_{x} \Psi_{x} d x=\int_{0}^{\pi}\left(\kappa\left(u_{x}+\varphi\right)+\left(\rho_{2}+\tau\right)\left(\varphi-\varphi^{*}\right)-\delta \theta-\rho_{2} \phi^{*}\right) \Psi d x, \forall \Psi \in H_{0}^{1}(0, \pi)$.

Thus, by virtue of the theory of elliptic equations, $\varphi \in H^{2}(0, \pi) \cap H_{*}^{1}(0, \pi)$ with

$$
\varphi_{x x}=\frac{-1}{b}\left(\kappa\left(u_{x}+\varphi\right)+\left(\rho_{2}+\tau\right)\left(\varphi-\varphi^{*}\right)-\delta \theta-\rho_{2} \phi^{*}\right) .
$$

Then, $\varphi$ solves 29$)_{2}$.

Substituting $u, \varphi, \theta$ just obtained in $(22),(24)$ and $(28)$, we infer that

$$
v \in H_{0}^{1}(0, \pi), \phi \in H_{*}^{1}(0, \pi) \text { and } \eta \in H_{\mu}^{1}\left((0,+\infty) ; H_{0}^{1}(0, \pi)\right) .
$$

Moreover, 26] implies that

$$
\int_{0}^{\infty} \mu(s) \eta_{x x}^{t}(s) d s \in L^{2}(0, \pi) .
$$

Finally we have

$$
\eta_{s}(s)=e^{-s} \theta+\eta^{*}(s)-\int_{0}^{s} e^{y-s} \eta^{*}(y) d y \in \mathcal{M}_{0}
$$

and $\eta(0)=0$, which proves that the solution $U$ of $(21)$ belongs to $D(\mathcal{A})$. Hence, Lumer-Phillips theorem ensures that the problem 20 has a unique solution $U(x, t)=$ $e^{\mathcal{A} t} U_{0}(x)$. This completes the proof of Theorem 3 .

\section{Asymptotic Behavior}

In this section we establish an exponential rate of decay for the solution of the system (16)-18. The following Lemma gives a sufficient condition for a $\mathrm{C}_{0}$-semigroup in order to be exponentially stable.

Lemma 2. [11] Let $S(t)$ be a contraction semigroup on $\mathcal{H}$, and let $\mathcal{A}$ be its infinitesimal generator. If the operator $i \beta I-\mathcal{A}$ is bounded below as $\beta \in \mathbb{R}$, that is there exists $\lambda>0$ such that

$$
\inf _{\beta \in \mathbb{R}}\|(i \beta I-\mathcal{A}) U\| \geq \lambda\|U\|, \forall U \in D(\mathcal{A}),
$$

then $S(t)$ is exponentially stable.

The main result of this paper reads as follows: 
Theorem 4. Assume that the memory kernel $\mu$ satisfies the hypotheses (h1)-(h5). Then the semigroup $S(t)=e^{\mathcal{A} t}$ associated to the problem (16)-(18) is exponentially stable.

Proof. The proof will be done by a contradiction argument. Suppose that the assertion is false. Then there exist a sequence $\left(\lambda_{n}\right) \subset \mathbb{R}$ and a sequence $\left(U_{n}\right) \subset$ $D(\mathcal{A})$, of unit norm

$$
\begin{aligned}
& \kappa\left\|D u_{n}+\varphi_{n}\right\|^{2}+\rho_{1}\left\|v_{n}\right\|^{2}+ b D \varphi_{n}\left\|^{2}+\rho_{2}\right\| \phi_{n}\left\|^{2}+c\right\| \theta_{n} \|^{2} \\
&+\int_{0}^{\infty} \mu(s)\left\|D \eta_{n}(s)\right\|^{2} d s=1,
\end{aligned}
$$

such that

$$
\lim _{n \longrightarrow \infty}\left\|\left(i \lambda_{n} I-\mathcal{A}\right) U_{n}\right\|=0
$$

which reads in components as

$$
\begin{gathered}
i \lambda_{n} u_{n}-v_{n} \longrightarrow 0 \text { in } H_{0}^{1}, \\
i \rho_{1} \lambda_{n} v_{n}-\kappa D^{2} u_{n}-\kappa D \varphi_{n}+\beta D \theta_{n} \longrightarrow 0 \text { in } L^{2}, \\
i \lambda_{n} \varphi_{n}-\phi_{n} \longrightarrow 0 \text { in } H_{*}^{1}, \\
i \rho_{2} \lambda_{n} \phi_{n}-b D^{2} \varphi_{n}+\kappa D u_{n}+\kappa \varphi_{n}+\tau \phi_{n}-\delta \theta_{n} \longrightarrow 0 \text { in } L_{*}^{2}, \\
i c \lambda_{n} \theta_{n}-\int_{0}^{\infty} \mu(s) D^{2} \eta_{n}(s) d s+\beta D v_{n}+\delta \phi_{n} \longrightarrow 0 \text { in } L^{2}, \\
i \lambda_{n} \eta_{n}-\theta_{n}+D_{s} \eta_{n} \longrightarrow 0 \text { in } \mathcal{M}_{1} .
\end{gathered}
$$

Note that since the norm in $\mathcal{H}$ is equivalent to the usual norm, then there exists $\gamma>0$ such that for any $U \in D(\mathcal{A})$ of unit norm, we have

$$
\left\|D u_{n}\right\|^{2}+\left\|v_{n}\right\|^{2}+\left\|\varphi_{n}\right\|^{2}+\left\|D \varphi_{n}\right\|^{2}+\left\|\phi_{n}\right\|^{2}+\left\|\theta_{n}\right\|^{2}+\int_{0}^{\infty} \mu(s)\left\|D \eta_{n}(s)\right\|^{2} d s=\gamma .
$$

First we have

$$
\operatorname{Re}\left\langle\left(i \lambda_{n} I-\mathcal{A}\right) U_{n}, U_{n}\right\rangle=\tau \int_{0}^{\pi} \phi_{n}^{2} d x-\frac{1}{2} \int_{0}^{\infty} \mu^{\prime}(s)\left\|D \eta_{n}(s)\right\|^{2} d s \longrightarrow 0 .
$$

Thus,

$$
\left\|\phi_{n}\right\| \longrightarrow 0
$$

and

$$
\left\|\eta_{n}\right\|_{\mathcal{M}_{1}}^{2} \leq-\frac{1}{\xi} \int_{0}^{\infty} \mu^{\prime}(s)\left\|D \eta_{n}(s)\right\|^{2} d s \longrightarrow 0 .
$$

Moreover, from (34) we have

$$
\varphi_{n} \sim \frac{1}{\lambda_{n}} \phi_{n} \longrightarrow 0 \text { in } L^{2} .
$$

The injection $L^{2} \hookrightarrow H^{-1}$ is continuous, hence 33 holds in $H^{-1}$ instead of $L^{2}$ and

$$
i \rho_{1} \lambda_{n} v_{n} \sim \kappa D^{2} u_{n}+\kappa D \varphi_{n}-\beta D \theta_{n} \text { in } H^{-1} \text {. }
$$


On the other hand we have

$$
\begin{gathered}
\left\|\kappa\left(D^{2} u_{n}+D \varphi_{n}\right)-\beta D \theta_{n}\right\|_{-1}=\sup _{\|D \psi\| \leq 1}\left|\left\langle\kappa\left(D^{2} u_{n}+D \varphi_{n}\right)-\beta D \theta_{n}, \psi\right\rangle\right| \\
\leq\left\|\kappa\left(D u_{n}+\varphi_{n}\right)-\beta \theta_{n}\right\| \cdot \sup _{\|D \psi\| \leq 1}\|D \psi\|, \\
\leq \kappa\left\|D u_{n}+\varphi_{n}\right\|+|\beta|\left\|\theta_{n}\right\| \leq \sqrt{2}
\end{gathered}
$$

Therefore,

$$
\left|\lambda_{n}\right|\left\|v_{n}\right\|_{-1} \leq C_{1}
$$

for a positive constant $C_{1}$ independent of $n \in \mathbb{N}$.

Similarly, we get

$$
\begin{aligned}
\left\|\int_{0}^{\infty} \mu(s) D^{2} \eta_{n}(s) d s\right\|_{-1} & \leq \int_{0}^{\infty} \mu(s)\left\|D \eta_{n}(s)\right\| d s \\
& \leq \sqrt{\int_{0}^{\infty} \mu(s) d s}\left(\int_{0}^{\infty} \mu(s) \int_{0}^{\pi}\left|D \eta_{n}\right|^{2}(s) d x d s\right)^{1 / 2}
\end{aligned}
$$

then

$$
\left\|\int_{0}^{\infty} \mu(s) D^{2} \eta_{n}(s) d s\right\|_{-1} \leq \sqrt{\int_{0}^{\infty} \mu(s) d s}\left\|\eta_{n}\right\|_{\mathcal{M}_{1}} \longrightarrow 0 .
$$

Note that 36 holds with $H^{-1}$ instead of $L^{2}$, hence

$$
\left\|i c \lambda_{n} \theta_{n}+\beta D v_{n}\right\|_{-1} \longrightarrow 0 \text {. }
$$

Since

$$
\left\|D v_{n}\right\|_{-1}=\sup _{\|D \psi\| \leq 1}\left|\left\langle D v_{n}, \psi\right\rangle\right| \leq\left\|v_{n}\right\|<\infty,
$$

$D v_{n}$ is bounded in $H^{-1}$, then

$$
\left\|c \lambda_{n} \theta_{n}\right\|_{-1} \leq C_{2}
$$

for a positive constant $C_{2}$ independent of $n \in \mathbb{N}$.

Next, we need to show that $\left\|\theta_{n}\right\| \longrightarrow 0$. Exploiting the continuous embedding of $\mathcal{M}_{1}$ into $\mathcal{M}_{0}, 37$ holds in $\mathcal{M}_{0}$ instead of $\mathcal{M}_{1}$. Let $\left(\xi_{n}\right)$ be the sequence $\xi_{n}=s \theta_{n}$. Clearly $\xi_{n} \in \mathcal{M}_{0}$. Indeed, from (h2), $\mu(s)$ goes to zero exponentially fast, then

$$
\int_{0}^{\infty} s^{2} \mu(s) \int_{0}^{\pi}\left|\theta_{n}\right|^{2} d x d s=\left\|\theta_{n}\right\|^{2} \int_{0}^{\infty} s^{2} \mu(s) d s=C_{3}<\infty .
$$

Multiplying (37) by $\xi_{n}$ in $\mathcal{M}_{0}$ we get

$$
\left\langle i \lambda_{n} \eta_{n}, \xi_{n}\right\rangle_{0}-\left\langle\theta_{n}, \xi_{n}\right\rangle_{0}+\left\langle D_{s} \eta_{n}, \xi_{n}\right\rangle_{0} \longrightarrow 0 .
$$

For the first term we have

$$
\left|\left\langle i \lambda_{n} \eta_{n}, \xi_{n}\right\rangle_{0}\right|=\left|\lambda_{n}\right| \int_{0}^{\infty} s \mu(s) \int_{0}^{\pi} \eta_{n} \theta_{n} d x d s .
$$


EXPONENTIAL STABILITY OF TIMOSHENKO SYSTEM WITH G-P THERMAL LAW 111

Then, using Hölder inequality we get

$$
\begin{aligned}
\left|\left\langle i \lambda_{n} \eta_{n}, \xi_{n}\right\rangle_{0}\right| & \leq\left|\lambda_{n}\right|\left\|\theta_{n}\right\|_{-1} \int_{0}^{\infty} s \mu(s)\left\|D \eta_{n}(s)\right\| d s \\
& \leq\left|\lambda_{n}\right|\left\|\theta_{n}\right\|_{-1} \sqrt{\int_{0}^{\infty} s^{2} \mu(s) d s} \int_{0}^{\infty} \mu(s)\left\|D \eta_{n}(s)\right\|^{2} d s, \\
& \leq C_{2} \sqrt{C_{3}}\left\|\eta_{n}\right\|_{1} \longrightarrow 0 .
\end{aligned}
$$

From (h4) we infer that $\lim _{s \rightarrow+\infty} s^{2} \mu(s)=0$, then, again (h4) and integration by parts yield

$$
-\int_{0}^{\infty} s^{2} \mu^{\prime}(s) d s=2 \int_{0}^{\infty} s \mu(s) d s=C_{4}<\infty .
$$

For the third term of 44 we have,

$$
\begin{aligned}
\left|\left\langle D_{s} \eta_{n}, \xi_{n}\right\rangle_{0}\right| & =\left|\int_{0}^{\infty} s \mu(s) \frac{d}{d s} \int_{0}^{\pi} \eta_{n} \theta_{n} d x d s\right| \\
& =\left|\int_{0}^{\infty} \mu(s) \int_{0}^{\pi} \eta_{n} \theta_{n} d x d s+\int_{0}^{\infty} s \mu^{\prime}(s) \int_{0}^{\pi} \eta_{n} \theta_{n} d x d s\right|,
\end{aligned}
$$

then,

$$
\begin{aligned}
\left|\left\langle D_{s} \eta_{n}, \xi_{n}\right\rangle_{0}\right| & \leq\left\|\theta_{n}\right\|\left[\int_{0}^{\infty} \mu(s)\left\|\eta_{n}\right\| d s-\int_{0}^{\infty} s \mu^{\prime}(s)\left\|\eta_{n}\right\| d s\right], \\
& \leq \int_{0}^{\infty} \mu(s)\left\|\eta_{n}\right\| d s-\int_{0}^{\infty} s \mu^{\prime}(s)\left\|\eta_{n}\right\| d s .
\end{aligned}
$$

Using the Cauchy-Schwarz and Poincaré's inequalities we conclude that

$$
\begin{aligned}
\int_{0}^{\infty} \mu(s)\left\|\eta_{n}\right\| d s & \leq \sqrt{\int_{0}^{\infty} \mu(s) d s} \sqrt{\int_{0}^{\infty} \mu(s)\left\|\eta_{n}\right\| d s} \\
& \leq \sqrt{\int_{0}^{\infty} \mu(s) d s}\left\|\eta_{n}\right\|_{0} \\
& \leq C_{P} \sqrt{\int_{0}^{\infty} \mu(s) d s}\left\|\eta_{n}\right\|_{1} \longrightarrow 0
\end{aligned}
$$

and

$$
\begin{aligned}
-\int_{0}^{\infty} s \mu^{\prime}(s)\left\|\eta_{n}\right\| d s & =\int_{0}^{\infty} s \sqrt{-\mu^{\prime}(s)} \sqrt{-\mu^{\prime}(s)}\left\|\eta_{n}\right\| d s \\
& \leq\left(-\int_{0}^{\infty} s^{2} \mu^{\prime}(s) d s\right)^{1 / 2}\left(-\int_{0}^{\infty} \mu^{\prime}(s)\left\|\eta_{n}\right\|^{2} d s\right)^{1 / 2} \\
& \leq\left(-C_{4} C_{P} \int_{0}^{\infty} \mu^{\prime}(s)\left\|D \eta_{n}\right\|^{2} d s\right)^{1 / 2} \longrightarrow 0
\end{aligned}
$$


Thus, (44) is reduced to

$$
\left\|\theta_{n}\right\|^{2} \int_{0}^{\infty} s \mu(s) d s=\left\langle\theta_{n}, \xi_{n}\right\rangle_{0} \longrightarrow 0,
$$

that is,

$$
\left\|\theta_{n}\right\|^{2}=\frac{2\left\langle\theta_{n}, \xi_{n}\right\rangle_{0}}{C_{4}} \longrightarrow 0 .
$$

Removing the terms that tend to 0 from (35), then multiplying by $\varphi_{n}$ we obtain

$$
i \rho_{2} \lambda_{n}\left\langle\phi_{n}, \varphi_{n}\right\rangle+b\left\|D \varphi_{n}\right\|^{2}+\kappa\left\langle D u_{n}, \varphi_{n}\right\rangle \longrightarrow 0 .
$$

We point out that

$$
\left\langle D u_{n}, \varphi_{n}\right\rangle \leq\left\|D u_{n}\right\|\left\|\varphi_{n}\right\| \longrightarrow 0
$$

and

Therefore,

$$
i \lambda_{n}\left\langle\phi_{n}, \varphi_{n}\right\rangle \sim\left\|\varphi_{n}\right\|^{2} \longrightarrow 0 .
$$

$$
\left\|D \varphi_{n}\right\| \longrightarrow 0 \text {. }
$$

Multiplying (32) by $\rho_{1} v_{n}$ and (33) by $u_{n}$ we get

$$
i \rho_{1} \lambda_{n}\left\langle u_{n}, v_{n}\right\rangle-\rho_{1}\left\|v_{n}\right\|^{2} \longrightarrow 0,
$$

and

$$
i \rho_{1} \lambda_{n}\left\langle v_{n}, u_{n}\right\rangle+\kappa\left\|D u_{n}\right\|^{2} \longrightarrow 0 .
$$

Adding (48) to the complex conjugate of (49), we get

$$
\kappa\left\|D u_{n}\right\|^{2}-\rho_{1}\left\|v_{n}\right\|^{2} \longrightarrow 0 .
$$

Combining (38), (39), 407, 41), 455), 477), and (50) we obtain

$$
\left(1+\frac{\rho_{1}}{\kappa}\right)\left\|v_{n}\right\|^{2} \rightarrow \gamma
$$

We complete the proof by showing that (51) leads to a contradiction.

Since $A^{-1} D v_{n}$ is bounded in $H_{0}^{1}$ (recall that $A=-D^{2}$ ), from 43 ) we have

$$
\left\langle i c \lambda_{n} \theta_{n}+\beta D v_{n}, A^{-1} D v_{n}\right\rangle=\left\langle i c \lambda_{n} \theta_{n}, A^{-1} D v_{n}\right\rangle+\beta\left\|D v_{n}\right\|_{-1}^{2} \longrightarrow 0 .
$$

On the other hand, from 45 we have

$$
\begin{aligned}
\left|\left\langle i c \lambda_{n} \theta_{n}, A^{-1} D v_{n}\right\rangle\right| & =\left|\left\langle i c \lambda_{n} \theta_{n}, A^{-1 / 2} v_{n}\right\rangle\right| \\
& \leq c\left|\lambda_{n}\right|\left\|A^{-1 / 2} v_{n}\right\|\left\|\theta_{n}\right\|=c\left|\lambda_{n}\right|\left\|v_{n}\right\|_{-1}\left\|\theta_{n}\right\| \\
& \leq c C_{1}\left\|\theta_{n}\right\| \longrightarrow 0 .
\end{aligned}
$$

Thus, (52) leads to

$$
\left\|D v_{n}\right\|_{-1} \longrightarrow 0
$$

From 19 we infer that

$$
\left\|v_{n}-\bar{v}_{n}\right\|=\left\|D v_{n}\right\|_{-1} \longrightarrow 0 .
$$


Therefore,

$$
\left\|v_{n}-\bar{v}_{n}\right\|^{2}=\left\|v_{n}\right\|^{2}-\pi\left|\bar{v}_{n}\right|^{2} \longrightarrow 0
$$

The comparison of (51) and (53) leads to

$$
\left|\bar{v}_{n}\right| \longrightarrow \sqrt{\frac{\kappa \gamma}{\pi\left(\kappa+\rho_{1}\right)}} .
$$

Thus, there exists a subsequence $\left(\bar{v}_{n}\right)$ that converges to $\bar{v}$, such that

$$
|\bar{v}|=\sqrt{\frac{\kappa \gamma}{\pi\left(\kappa+\rho_{1}\right)}} .
$$

Using (53) again we conclude that there exists a subsequence of $\left(v_{n}\right)$ which converges to $\bar{v}$ in $L^{2}(0, \pi)$. Exploiting the continuous embedding of $L^{2}(0, \pi)$ into $H^{-1}(0, \pi)$, one can deduce that

$$
v_{n} \longrightarrow \bar{v}, \quad \text { in } H^{-1}(0, \pi) .
$$

At this point we distinguish two cases. Suppose that $\left(\lambda_{n}\right)$ is unbounded, then we can choose a subsequence $\left(\lambda_{n}\right)$ such that $\left|\lambda_{n}\right| \longrightarrow \infty$ and from (42) we have

$$
v_{n} \longrightarrow 0 \text { in } H^{-1}(0, \pi) \text {. }
$$

From the uniqueness of the limit we conclude that $\bar{v}=0$, which is incompatible with (54).

Conversely, assume that $\left(\lambda_{n}\right)$ is bounded, again, there exists a subsequence $\left(\lambda_{n}\right)$ that converges to some $\lambda \in \mathbb{R}$. In this case we have

$$
\lim _{n \longrightarrow \infty}\left\|(i \lambda I-\mathcal{A}) U_{n}\right\|=0
$$

and (32)-37) hold with $\lambda$ instead of $\lambda_{n}$. In particular

$$
i \lambda u_{n}-v_{n} \longrightarrow 0 \text { in } H_{0}^{1}(0, \pi) \text {. }
$$

Since $\left(u_{n}\right)$ is bounded in $H_{0}^{1}(0, \pi)$, we conclude that there exists $v^{*} \in H_{0}^{1}(0, \pi)$ and a subsequence $\left(v_{n}\right)$ that converges weakly to $v^{*}$ in $H_{0}^{1}(0, \pi)$. From the uniqueness of the limit we infer that $v^{*}=\bar{v}$, which is in contradiction with $v^{*} \in H_{0}^{1}(0, \pi)$, since $\bar{v}$ is a non-zero constant function, and therefore cannot be in $H_{0}^{1}(0, \pi)$. This completes the proof of Theorem 4

Declaration of Competing Interests The author declare that he has no conflict of interest.

Acknowledgements The author expresses his sincere thanks to the anonymous reviewers for their comments and remarks which improve the presentation of this paper. The present work is supported by the General Directorate of Scientific Research and Technological Development (DGRSDT) Algeria, PRFU project \# C00L03UN390120220004. 


\section{REFERENCES}

[1] Almeida Jùnior, D. S., Santos, M. L., Muñoz Rivera, J. E., Stability to 1-D thermoelastic Timoshenko beam acting on shear force, Z. Angew. Math. Phys., 65 (2014), 1233-1249. https://doi.org/10.1007/s00033-013-0387-0

[2] Alves, M. S., Jorge Silva, M. A., Ma, T. F., Muñoz Rivera, J. E., Invariance of decay rate with respect to boundary conditions in thermoelastic Timoshenko systems, Z. Angew. Math. Phys., 67 (2016), 70. https://doi.org/ 10.1007/s00033-016-0662-y

[3] Alves, M. S., Jorge Silva, M. A., Ma, T. F., Muñoz Rivera, J. E., Non-homogeneous thermoelastic Timoshenko systems, Bull. Braz. Math. Soc. (N. S.), 48 (2017), 461-484. https://doi.org/10.1007/s00574-017-0030-3

[4] Apalara, T. A., Uniform stability of a laminated beam with structural damping and second sound, Z. Angew. Math. Phys., 68(2) (2017). https://doi.org/10.1007/s00033-017-0784-x

[5] Choucha, A., Ouchenane, D., Boulaaras, S., Well posedness and stability result for a thermoelastic laminated Timoshenko beam with distributed delay term, Math. Meth. Appl. Sci., 43(17) (2020), 1-22.https://doi.org/10.1002/mma.6673

[6] Choucha, A., Boulaaras, S., Ouchenane, D., Alkhalaf, S., Stability result and well posedness for Timoshenko's beam laminated with termoelastic and past history, Fractals, 29 (2021), 1-26. https://doi.org/10.1142/S0218348X21400259

[7] Dell'Oro, F., Pata, V., On the stability of Timoshenko systems with Gurtin-Pipkin thermal law, J. Diff. Equa., 257 (2014), 523-548. https://doi.org/10.1016/j.jde.2014.04.009

[8] Fatori, L. H., Muñoz Rivera, J. E., Energy decay for hyperbolic thermoelastic systems of memory type, Quart. Appl. Math., 59 (2001), 441-458. https://doi.org/10.1090/qam/1848527

[9] Fatori, L. H., Muñoz Rivera, J. E., Monteiro, R. N., Energy decay to Timoshenko's system with thermoelasticity of type III, Asymptotic Analysis, 86 (2014), 227-247.

[10] Fernàndez-Sare H. D., Racke, R., On the stability of damped Timoshenko system Cattaneo versus Fourier law, Arch. Rat. Mech. Anal., 194 (2009), 221-251. https://doi.org/10.1007/s00205-009-0220-2

[11] Giorgi, C., Naso, M. G., Pata, V., Exponential stability in linear heat conduction with memory: a semigroup approach, Commun. Appl. Anal., 5 (2001), 121-134.

[12] Green, A. E., Naghdi, P. M., A re-examination of the basic postulates of thermomechanics, Proc. Roy. Soc. London Ser., A432 (1991), 171-194. https://doi.org/10.1098/rspa.1991.0012

[13] Green, A. E., Naghdi, P. M., On undamped heat waves in an elastic solid, J. Thermal Stresses, 15 (1992), 253-264. https://doi.org/10.1080/01495739208946136

[14] Green, A. E., Naghdi, P. M., Thermoelasticity without energy-dissipation. J. Elast., 31(3) (1993), 189-208. https://doi.org/10.1007/BF00044969

[15] Grobbelaar-Van Dalsen, M., Strong stabilization of models incorporating the thermoelastic Reissner-Mindlin plate equations with second sound, Applicable Analysis, 90(9) (2011), 14191449. https://doi.org/10.1080/00036811.2010.530259

[16] Gurtin, M. E., Pipkin, A. C., A general theory of heat conduction with finite wave speeds, Arch. Ration. Mech. Anal., 31 (1968),113-126. https://doi.org/10.1007/BF00281373

[17] Guesmia, A., Messaoudi, S. A., Wahbe, A., Uniform decay in mildly damped Timoshenko systems with non-equal wave speed propagation, Dynamic Systems and Applications, 21 (2012), 133-146. https://hal.inria.fr/hal-01281866

[18] Jorge Silva, M. A., Pinheiro, S. B., Improvement on the polynomial stability for a Timoshenko system with type III thermoelasticity, Applied Mathematics Letters, 96 (2019), 95100. https://doi.org/10.1016/j.aml.2019.04.014

[19] Jorge Silva, M. A., Racke, R., Effects of history and heat models on the stability of thermoelastic Timoshenko systems, J. Diff. Equ., 275 (2021), 167-203. https://doi.org/10.1016/j.jde.2020.11.041 
[20] Liu, W. J., Zhao, W., Exponential and polynomial decay for a laminated beam with Fourier's type heat conduction, Preprints, (2017) https://doi.org/10.20944/preprints201702.0058.v2

[21] Lord H. W., Shulman, Y., A generalized dynamical theory of thermoelasticity, J. Mech. Phys. Sol., 15 (1967), 299-309. https://doi.org/10.1016/0022-5096(67)90024-5

[22] Messaoudi, S. A., Pokojovy, M., Said-Houari, B., Nonlinear damped Timoshenko systems with second: global existence and exponential stability, Math. Method. Appl. Sci., 32 (2009), 505-534. https://doi.org/10.1002/mma.1049.

[23] Messaoudi, S. A., Fareh, A., Energy decay in a Timoshenko-type system of thermoelasticity of type III with different wave-propagation speeds, Arab J Math., 2 (2013), 199-207. https://doi.org/10.1007/s40065-012-0061-y

[24] Messaoudi, S. A., Said-Houari, B., Energy decay in a Timoshenko-type system of thermoelasticity of type III. J. Math. Anal. Appl., 348 (2008), 298-307. https://doi.org/10.1016/j.jmaa.2008.07.036

[25] Muñoz Rivera, J. E., Racke, R., Mildly dissipative nonlinear Timoshenko systemsglobal existence and exponential stability, J. Math. Anal. Appl., 276 (2002), 248-278. https://doi.org/10.1016/S0022-247X(02)00436-5

[26] Pata, V., Vuk, E., On the exponential stability of linear thermoelasticity, Contin. Mech. Thermodyn., 12 (2000), 121-130. https://doi.org/doi:10.1007/s001610050131

[27] Pazy, A., Semigroups of Linear Pperators and Applications to Partial Differential Equations, Springer-Verlag, New York, 1983.

[28] Quintanilla, R., Racke, R., Qualitative aspects of solutions in resonators, Arch. Mech., 60(4) (2008), 345-360.

[29] Raposo, C. A., Exponential stability for a structure with interfacial slip and frictional damping, Applied Mathematics Letters, 53 (2016), 85-91. https://doi.org/10.1016/j.aml.2015.10.005

[30] Santos, M. L., Almeida Jünior, D. S., On Timoshenko-type systems with type III thermoelasticity: asymptotic behavior, J. Math. Appl., 448 (2017), 650-671. https://doi.org/10.1016/j.jmaa.2016.10.074

[31] Santos, M. L., Almeida Jünior, D. S., Muñoz Rivera, J. E., The stability number of the Timoshenko system with second sound, J. Differential Equations, 253 (2012), 2715-2733. https://doi.org/10.1016/j.jde.2012.07.012.

[32] Timoshenko, S. P., On the correction for shear of the differential equation for transverse vibrations of bars of Prismatic bars, Dubl. Philos. Mag., 41 (1921), 744-746. https://doi.org/10.1080/14786442108636264

[33] Vrabie, I. I., C C-Semigroups and Applications, Elsevier Science B.V., Amesterdam, 2003. 